\title{
Soluble dietary fibre (pectin) increases satiety and decreases adiposity in fat rats on a high fat diet
}

\author{
C. L. Adam, L. M. Thomson, P. A. Williams and A. W. Ross \\ Obesity \& Metabolic Health Division, Rowett Institute of Nutrition \& Health, University of Aberdeen, Aberdeen AB21 \\ $9 S B$, Scotland, UK
}

Macronutrient-induced satiety may provide a natural countermeasure to obesity and one such macronutrient is indigestible complex carbohydrate or soluble dietary fibre. We have previously demonstrated decreased voluntary food intake and decreased adiposity in conventional adult rats fed different types of soluble dietary fibre, including pectin ${ }^{(1)}$. This study tested the hypothesis that soluble dietary fibre (pectin) may also increase satiety and decrease adiposity in high fat diet-induced obese rats.

In two independently replicated experiments, adult 12-week old male outbred Sprague Dawley rats that had previously been reared on a high fat diet (standard purified $45 \% \mathrm{kcal}$ fat diet; HF) were offered the following purified experimental diets ad libitum for 4 weeks ( $n 8$ per experiment per group): low fat diet (AIN-93M diet, 10\% kcal fat), low fat-high fibre diet (AIN-93M with 10\% w/ w pectin), high fat diet (HF) or high fat-high fibre diet (HF with 10\% w/w pectin); all diets were supplied by Special Diet Services Ltd. (Witham, Essex, UK). Voluntary food intake was measured daily, body weight recorded twice weekly, MRI scans performed at start and end to determine body composition, and final trunk blood samples were obtained. Radioimmunoassay kits were used to determine plasma hormone concentrations (Merck Millipore, Billerica, MA, USA). Mean (and range) body weight and total body fat content at the start were $542(420-630) \mathrm{g}$ and $13(8-24) \%$, respectively, and final results after 4 weeks were:

\begin{tabular}{|c|c|c|c|c|c|c|c|c|}
\hline & \multicolumn{8}{|c|}{ Diet ( $n 16$ per group) } \\
\hline & \multicolumn{2}{|l|}{ Low fat } & \multicolumn{2}{|c|}{ Low fat + pectin } & \multicolumn{2}{|c|}{ High fat } & \multicolumn{2}{|c|}{ High fat + pectin } \\
\hline & Mean & SEM & $\overline{\text { Mean }}$ & $\overline{\text { SEM }}$ & Mean & $\overline{\text { SEM }}$ & Mean & SEM \\
\hline Cumulative caloric intake (kcal) & $2541 \cdot 9^{\mathrm{a}}$ & $317 \cdot 7$ & $2012 \cdot 0^{\mathrm{b}}$ & $188 \cdot 7$ & $2849 \cdot 1^{\mathrm{c}}$ & $263 \cdot 6$ & $2337 \cdot 6^{\mathrm{a}}$ & $259 \cdot 1$ \\
\hline Bodyweight gain (g) & $70 \cdot 24^{\mathrm{a}}$ & $6 \cdot 03$ & $20 \cdot 01^{\mathrm{b}}$ & $5 \cdot 74$ & $98 \cdot 02^{\mathrm{c}}$ & $4 \cdot 83$ & $20 \cdot 83^{\mathrm{b}}$ & $4 \cdot 83$ \\
\hline Total fat mass change $(\mathrm{g})$ & $3.63^{\mathrm{a}}$ & 3.70 & $-26 \cdot 18^{\mathrm{b}}$ & $2 \cdot 78$ & $28 \cdot 46^{\mathrm{c}}$ & 1.60 & $-13 \cdot 53^{d}$ & $3 \cdot 26$ \\
\hline Final total body fat $(\%)$ & $11 \cdot 7^{\mathrm{a}}$ & 1.01 & $7 \cdot 81^{\mathrm{b}}$ & $0 \cdot 56$ & $16 \cdot 11^{\mathrm{c}}$ & $0 \cdot 79$ & $9 \cdot 47^{\mathrm{ab}}$ & $0 \cdot 61$ \\
\hline Plasma leptin (ng/ml) & $9 \cdot 36^{\mathrm{a}}$ & $1 \cdot 11$ & $4.97^{\mathrm{b}}$ & 0.58 & $14 \cdot 67^{\mathrm{c}}$ & $1 \cdot 10$ & $5 \cdot 62^{\mathrm{b}}$ & 0.60 \\
\hline Plasma insulin $(\mathrm{ng} / \mathrm{ml})$ & $1.59^{\mathrm{a}}$ & $0 \cdot 14$ & $0 \cdot 96^{\mathrm{b}}$ & $0 \cdot 14$ & $1.79^{\mathrm{a}}$ & $0 \cdot 16$ & $0.78^{\mathrm{b}}$ & 0.07 \\
\hline Plasma PYY (pg/ml) & $325 \cdot 1^{\mathrm{a}}$ & $38 \cdot 0$ & $506 \cdot 7^{\mathrm{b}}$ & $51 \cdot 6$ & $355 \cdot 2^{\mathrm{a}}$ & $38 \cdot 9$ & $505 \cdot 3^{\mathrm{b}}$ & $42 \cdot 2$ \\
\hline
\end{tabular}

Within rows, mean values with different superscript letters are significantly different, $P<0.05$ (ANOVA).

Caloric intake, bodyweight gain, final adiposity, plasma leptin and insulin were all lower, and plasma satiety hormone PYY was higher, in rats given low fat + pectin diet than low fat diet alone and in rats given high fat + pectin diet than high fat diet alone. Whereas body fat mass increased during the experiment on high fat diet, and was maintained on low fat diet, significant body fat loss occurred in both pectin-fed groups.

Therefore, even if a high fat diet is consumed, the addition of soluble fibre leads to increased satiety and decreased caloric intake, adiposity, leptinaemia and insulinaemia, indicating its potential for weight loss and improving metabolic phenotype in diet-induced obesity.

Research funded by the Scottish Government Rural and Environment Science and Analytical Services Division (RESAS).

1. Adam CL, Findlay PA, Thomson LM, Ross AW (2012) Appetite 59, e2. 